MATHEMATICS OF COMPUTATION

Volume 70, Number 235, Pages 1221-1235

S $0025-5718(00) 01227-8$

Article electronically published on February 23, 2000

\title{
POWER SERIES EXPANSIONS FOR MATHIEU FUNCTIONS WITH SMALL ARGUMENTS
}

\author{
G. C. KOKKORAKIS AND J. A. ROUMELIOTIS
}

\begin{abstract}
Power series expansions for the even and odd angular Mathieu functions $\operatorname{Se}_{m}(h, \cos \theta)$ and $\operatorname{So}_{m}(h, \cos \theta)$, with small argument $h$, are derived for general integer values of $m$. The expansion coefficients that we evaluate are also useful for the calculation of the corresponding radial functions of any kind.
\end{abstract}

\section{INTRODUCTION}

Mathieu functions are used in many applications in engineering and physics 1]-12 originating, principally, from the solution of the wave equation in elliptic cylindrical coordinates. Studies on these functions can be found in [13]-17], among other places. Numerical computation of Mathieu functions with integer orders is examined in [18]-25].

In this paper we produce power series expansions for the even and odd angular Mathieu functions $\operatorname{Se}_{m}(h, \cos \theta)$ and $\operatorname{So}_{m}(h, \cos \theta)$, with small argument $h$, for general integer values of $m$. In [13]- 16], [26], [27] the lower order expansion coefficients were given only for the special values $m=0, \ldots, 4$.

The expansion coefficients that we calculate here can also be used to evaluate the corresponding radial Mathieu functions of any kind.

An analogous method was used in [28] for the evaluation of the spheroidal wave functions.

The calculation of the expansion coefficients for the even and odd functions is developed in Sections 2 and 3, respectively, while in Section 4 we give some relations connecting the various expansion coefficients of the even and odd functions. Finally, in Section 5 we compare our results, for special values of the parameters, with previous ones produced by other methods. These comparisons confirm the correctness of our formulas, at least for those values of the parameters, giving a very good check on the validity of our procedure.

\section{Calculation of the expansion coefFicients FOR THE EVEN FUNCTIONS}

The angular Mathieu functions are solutions of Mathieu's differential equation

$$
\frac{d^{2} y}{d \theta^{2}}+\left(b-\frac{h^{2}}{2} \cos 2 \theta\right) y=0
$$

Received by the editor May 19, 1998 and, in revised form, April 13, 1999 and July 8, 1999. 2000 Mathematics Subject Classification. Primary 33E10. 
where $b$ and $h^{2}$ are constants. These functions are periodic, even $\left(\mathrm{Se}_{m}\right)$ or odd $\left(\mathrm{So}_{m}\right)$, only for certain discrete characteristic values (eigenvalues) of $b$, with symbols $b e_{m}$ or $b o_{m}$, respectively, and $m$ an integer. In this section we examine the even functions, which are given by the series [13]-[15],

$$
\mathrm{Se}_{m}(h, \cos \theta)=\sum_{n=0}^{\infty} B_{n}^{e}(h, m) \cos n \theta, \quad m \geq 0 .
$$

Here $m$ and $n$ are both even or both odd. Substituting from (2) into (11), we obtain the following second order recurrence relation for the expansion coefficients $B_{n}^{e}(h, m)$ [13]-[15], [17]:

$$
\left(-n^{2}+b e_{m}\right) B_{n}^{e}-\frac{h^{2}}{4}\left[B_{n+2}^{e}+\frac{2}{\varepsilon_{n-2}} B_{n-2}^{e}+\left(2-\varepsilon_{n-1}\right) B_{n}^{e}\right]=0, \quad m, n \geq 0,
$$

where $\varepsilon_{0}=1$ and $\varepsilon_{i}=2$ for $i \neq 0$ is the Neumann factor, and $B_{-n}^{e}=0$ for $n>0$.

When $h \rightarrow 0, \operatorname{Se}_{m}(h, \cos \theta) \rightarrow \cos m \theta$ and $b e_{m} \rightarrow m^{2}$. In this case, the only nonzero coefficient is $B_{m}^{e}(h, m) \rightarrow 1$.

Approximations for $S e_{m}$, valid for small values of $h$, follow by expanding $B_{n}^{e}(h, m)$ in power series in $h$. Keeping in mind the above remarks, we see that this expansion must be of the form

$$
\begin{gathered}
B_{m \pm 2 q}^{e}(h, m)=\left[\alpha_{2 q, 0}^{ \pm} h^{2 q}+\alpha_{2 q, 2}^{ \pm} h^{2 q+2}+\alpha_{2 q, 4}^{ \pm} h^{2 q+4}+\cdots\right] B_{m}^{e}(h, m), \\
m \geq 0(+), \quad m \geq 2 q(-), \quad q=0,1,2, \ldots,
\end{gathered}
$$

where $\alpha_{2 q, 2 k}^{-}=0(k \geq 0)$ if $0 \leq m<2 q$, while $\alpha_{0,0}^{ \pm}=1$ and $\alpha_{0,2 k}^{ \pm}=0$ if $k \geq 1$.

The expansion for $b e_{m}(h)$ must be

$$
b e_{m}(h)=m^{2}+l_{2} h^{2}+l_{4} h^{4}+l_{6} h^{6}+\cdots .
$$

In (4), (5), and in what follows we omit the indices $m$ and $e$ from the various expansion coefficients $\alpha$ and $l$, as well as from any others that may be used, for reasons of simplicity. Certainly, these coefficients are independent of $h$.

By substituting (4) and (5) into (3) and equating the coefficients of $h^{2}, h^{4}, \ldots$ to zero separately, we obtain with $n=m \geq 0$, first from the coefficient of $h^{2}$

$$
l_{2}=\left(2-\varepsilon_{m-1}\right) / 4
$$

and next from that of $h^{2 k+4}$

$$
\frac{1}{4}\left(\alpha_{2,2 k}^{+}+\frac{2}{\varepsilon_{m-2}} \alpha_{2,2 k}^{-}\right)=l_{2 k+4}, \quad k=0,1,2, \ldots
$$

Setting now $n=m \pm 2 q$, where $q \geq 1$ ( $m \geq 2 q$ for the lower sign), in (3) and using (4) and (5), we obtain by equating to zero, first the coefficient of $h^{2 q}$

$$
\alpha_{2 q, 0}^{ \pm}=f^{ \pm}(2 q-2) \alpha_{2 q-2,0}^{ \pm}, \quad q \geq 1,
$$

next that of $h^{2 q+2}$

$$
\alpha_{2 q, 2}^{ \pm}=f^{ \pm}(2 q-2) \alpha_{2 q-2,2}^{ \pm}+g^{ \pm}(2 q) \alpha_{2 q, 0}^{ \pm}, \quad q \geq 1,
$$


and finally that of $h^{2 q+2 k}$

$$
\begin{aligned}
\alpha_{2 q, 2 k}^{ \pm}= & f^{ \pm}(2 q-2) \alpha_{2 q-2,2 k}^{ \pm}+g^{ \pm}(2 q) \alpha_{2 q, 2 k-2}^{ \pm}+t^{ \pm}(2 q) \sum_{j=2}^{k} l_{2 j} \alpha_{2 q, 2 k-2 j}^{ \pm} \\
& +p^{ \pm}(2 q+2) \alpha_{2 q+2,2 k-4}^{ \pm}, \quad q \geq 1, k \geq 2,
\end{aligned}
$$

where we have made the substitutions $(q \geq 1)$

$$
\begin{gathered}
t^{ \pm}(2 q)= \pm \frac{1}{4 q(m \pm q)}, \\
f^{+}(2 q-2)=-\frac{1}{2 \varepsilon_{m+2 q-2}} t^{+}(2 q), \quad f^{-}(2 q-2)=-\frac{1}{4} t^{-}(2 q), \\
g^{+}(2 q)=\frac{2-\varepsilon_{m-1}}{4} t^{+}(2 q), \quad g^{-}(2 q)=-\frac{2-\varepsilon_{m-2 q-1}}{4} t^{-}(2 q), \\
p^{+}(2 q+2)=-\frac{1}{4} t^{+}(2 q), \quad p^{-}(2 q+2)=-\frac{1}{2 \varepsilon_{m-2 q-2}} t^{-}(2 q) .
\end{gathered}
$$

From (9) with $q=1$ we obtain

$$
\alpha_{2,2}^{ \pm}=g^{ \pm}(2) \alpha_{2,0}^{ \pm}=v_{2}^{ \pm}(2) \alpha_{2,0}^{ \pm},
$$

where

$$
v_{2}^{ \pm}(2)=g^{ \pm}(2)
$$

Setting, for $q \geq 2$,

$$
\alpha_{2 q, 2}^{ \pm}=v_{2}^{ \pm}(2 q) \alpha_{2 q, 0}^{ \pm},
$$

we get from (8), (9), (17) that

$$
\alpha_{2 q, 2}^{ \pm}=\left[v_{2}^{ \pm}(2 q-2)+g^{ \pm}(2 q)\right] \alpha_{2 q, 0}^{ \pm} .
$$

From (17) and (18) we get the recurrence relation

$$
v_{2}^{ \pm}(2 q)=v_{2}^{ \pm}(2 q-2)+g^{ \pm}(2 q), \quad q \geq 1,
$$

where $v_{2}^{ \pm}(0)=0$.

Using (13), (16), (19), we obtain

$$
v_{2}^{+}(2 q)=\sum_{i=1}^{q} g^{+}(2 i)=\left\{\begin{array}{l}
\frac{1}{16} \sum_{i=1}^{q}\left(\frac{1}{i}-\frac{1}{i+1}\right)=\frac{q}{16(q+1)}, \quad m=1, \\
0, \quad m \neq 1,
\end{array}\right.
$$

for $q \geq 0$, and, for $q \geq 1$,

$$
v_{2}^{-}(2 q)=\sum_{i=1}^{q} g^{-}(2 i)=\left\{\begin{array}{l}
\frac{1}{16 q(q+1)}, \quad m=2 q+1, \\
0, \quad m \neq 2 q+1 .
\end{array}\right.
$$

In (20b) $g^{-}(2 i) \neq 0$ only for $m=2 i+1$, and as $m \geq 2 q$ in this case, the only value of $i$ giving a nonzero result is $i=q$.

Setting now

$$
\alpha_{2 q, 2 k}^{ \pm}=v_{2 k}^{ \pm}(2 q) \alpha_{2 q, 0}^{ \pm}
$$


for $q \geq 1$ and $k \geq 2$, we find from (8), (10) and (21) that

$$
\begin{aligned}
\alpha_{2 q, 2 k}^{ \pm}= & {\left[v_{2 k}^{ \pm}(2 q-2)+g^{ \pm}(2 q) v_{2 k-2}^{ \pm}(2 q)+t^{ \pm}(2 q) \sum_{j=2}^{k} l_{2 j} v_{2 k-2 j}^{ \pm}(2 q)\right.} \\
& \left.+p^{ \pm}(2 q+2) v_{2 k-4}^{ \pm}(2 q+2) f^{ \pm}(2 q)\right] \alpha_{2 q, 0}^{ \pm} \quad(q \geq 1, k \geq 2) .
\end{aligned}
$$

It is evident from (21) that $v_{0}^{ \pm}(2 q)=1$. From (21), (22) we obtain the following recurrence relation for the calculation of $v_{2 k}^{ \pm}(2 q)$ :

$$
\begin{array}{r}
v_{2 k}^{ \pm}(2 q)=v_{2 k}^{ \pm}(2 q-2)+g^{ \pm}(2 q) v_{2 k-2}^{ \pm}(2 q)+t^{ \pm}(2 q) \sum_{j=2}^{k} l_{2 j} v_{2 k-2 j}^{ \pm}(2 q) \\
+p^{ \pm}(2 q+2) v_{2 k-4}^{ \pm}(2 q+2) f^{ \pm}(2 q) \quad(q \geq 1, k \geq 2) .
\end{array}
$$

From (23) with $q=1$ we get that

$$
v_{2 k}^{ \pm}(2)=g^{ \pm}(2) v_{2 k-2}^{ \pm}(2)+t^{ \pm}(2) \sum_{j=2}^{k} l_{2 j} v_{2 k-2 j}^{ \pm}(2)+p^{ \pm}(4) v_{2 k-4}^{ \pm}(4) f^{ \pm}(2),
$$

where we have used the result $v_{2 k}^{ \pm}(0)=0$ for $k \geq 1$.

From (23), (24) we find that

$$
\begin{aligned}
& v_{2 k}^{ \pm}(2 q)=\sum_{i=1}^{q}\left[g^{ \pm}(2 i) v_{2 k-2}^{ \pm}(2 i)+t^{ \pm}(2 i) \sum_{j=2}^{k} l_{2 j} v_{2 k-2 j}^{ \pm}(2 i)\right. \\
&\left.+p^{ \pm}(2 i+2) v_{2 k-4}^{ \pm}(2 i+2) f^{ \pm}(2 i)\right] \\
&=\beta_{2 k}^{ \pm}(2 q)+\sum_{j=2}^{k} l_{2 j} \gamma_{2 k-2 j}^{ \pm}(2 q)+\delta_{2 k}^{ \pm}(2 q), \quad q \geq 1, k \geq 2,
\end{aligned}
$$

where

$$
\begin{gathered}
\beta_{2 k}^{ \pm}(2 q)=\sum_{i=1}^{q} g^{ \pm}(2 i) v_{2 k-2}^{ \pm}(2 i), \quad k \geq 1, \\
\gamma_{2 k-2 j}^{ \pm}(2 q)=\sum_{i=1}^{q} t^{ \pm}(2 i) v_{2 k-2 j}^{ \pm}(2 i), \quad k \geq j \geq 2, \\
\delta_{2 k}^{ \pm}(2 q)=\sum_{i=1}^{q} p^{ \pm}(2 i+2) v_{2 k-4}^{ \pm}(2 i+2) f^{ \pm}(2 i), \quad k \geq 2 .
\end{gathered}
$$

Formula (28) for $\delta_{2 k}^{-}(2 q)$ is correct for $m \geq 2 q+2$. For $m=2 q+1$ or $m=2 q$ the term with $i=q$ should be omitted from the sum because it corresponds to the expansion coefficient $\alpha_{2 q+2,2 k-4}^{-}$(see (10), (22), (23), (25), (28)), which is equal to zero for $m<2 q+2$. The sum in the last two aforementioned cases, extended from $i=1$ to $i=q-1$, is considered as zero if its upper limit is less than 1 , i.e., for $q=1$. 
The recurrence relation (25) can be used for the evaluation of $v_{2 k}^{ \pm}(2 q)$, and consequently of $\alpha_{2 q, 2 k}^{ \pm}$from (21), by using the expressions of $v_{2 k-2 s}^{ \pm}(2 i)(i \geq 1,1 \leq s \leq k)$. The expansion coefficients $l_{2 j}(2 \leq j \leq k)$ are calculated from (7) by using $\alpha_{2,2 j-4}^{ \pm}$ calculated from (21) with $q=1$. Their expressions for $j \leq 8$ can be found also in [13], [15], [17].

For great values of $k, v_{2 k}^{ \pm}(2 q)$ can easily be obtained numerically from (25). The process must be repeated for each different value of $m$ and $q$. However, for small values of $k$, analytic closed-form expressions are obtained, valid for each $m$ and $q$. So, for $k=2$, we find from (25) that

$$
v_{4}^{ \pm}(2 q)=\beta_{4}^{ \pm}(2 q)+l_{4} \gamma_{0}^{ \pm}(2 q)+\delta_{4}^{ \pm}(2 q) .
$$

Substituting (13) and (20) into (26), we obtain

$$
\begin{aligned}
& \beta_{4}^{+}(2 q)= \begin{cases}\frac{1}{256} \sum_{i=1}^{q} \frac{1}{(i+1)^{2}}, & m=1, \\
0, & m \neq 1,\end{cases} \\
& \beta_{4}^{-}(2 q)= \begin{cases}\frac{1}{256 q^{2}(q+1)^{2}}, & m=2 q+1, \\
0, & m \neq 2 q+1 .\end{cases}
\end{aligned}
$$

In (30b) the remarks made after (20b) have been used.

Next, substituting (11) into (27) with $k=j=2$, we obtain

$$
\gamma_{0}^{ \pm}(2 q)= \pm \sum_{i=1}^{q} \frac{1}{4 i(m \pm i)} .
$$

Finally, substituting (12) and (14) into (28), with $k=2$, we obtain

$$
\begin{aligned}
& \delta_{4}^{+}(2 q)= \frac{1}{256} \sum_{i=1}^{q} \frac{1}{i(i+1)(i+m)(i+m+1)}, \\
& \delta_{4}^{-}(2 q)=\left\{\begin{array}{cc}
\frac{1}{256} \sum_{i=1}^{q} \frac{1}{i(i+1)(i-m)(i-m+1)}, \quad m \geq 2 q+3, \\
\frac{1}{256} \sum_{i=1}^{q-1} \frac{1}{i(i+1)(i-m)(i-m+1)} \\
\frac{1}{128 q(q+1)(q-m)(q-m+1)}, \quad m=2 q+2, \\
\frac{1}{256} \sum_{i=1}^{q-1} \frac{1}{i(i+1)(i-m)(i-m+1)}, \quad m=2 q+1, \\
\frac{1}{256} \sum_{i=1}^{q-2} \frac{1}{i(i+1)(i-m)(i-m+1)} \\
\quad+\frac{1}{128(q-1) q(q-1-m)(q-m)}, \quad m=2 q, q \neq 1, \\
0, \quad m=2(q=1) .
\end{array}\right.
\end{aligned}
$$

In the sums of (32b) we have used the remarks after (28). Also, for $m=2 q+2$ or $m=2 q$ their last term (with $i=q$ or $i=q-1$, respectively) is written separately, because in these cases the Neumann factor appearing in (14) is equal to 1, while in the rest it is equal to 2 . Sums whose upper limit is less than 1 should be taken as zero.

We expand the fractions after the summation signs in (31) and (32) into sums of partial fractions, and we next substitute (30)-(32) in (29) by using the expressions 
for $l_{4}$ calculated from (77) and (8) with $k=0$ and $q=1$, respectively:

$$
l_{4}=\left\{\begin{array}{l}
-1 / 128, \quad m=1, \\
5 / 192, \quad m=2, \\
1 /\left[32\left(m^{2}-1\right)\right], \quad m=0 \text { or } m \geq 3 .
\end{array}\right.
$$

So, we finally obtain, after straightforward but lengthy calculations, the results

$$
v_{4}^{+}(2 q)=\left\{\begin{array}{l}
\frac{q}{1024(q+2)}, \quad m=1, \\
\frac{q\left(47 q^{2}+222 q+247\right)}{9216(q+1)(q+2)(q+3)}, \quad m=2, \\
\frac{q\left(m^{2}+3 m+m q+3 q+4\right)}{256\left(m^{2}-1\right)(m+1)(q+1)(m+q+1)}, \quad m=0 \text { or } m \geq 3,
\end{array}\right.
$$

for $q \geq 0$, and, for $q \geq 1$,

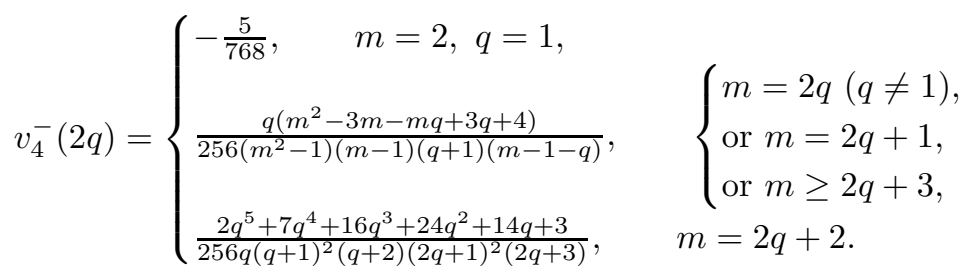

From (8), [11), (12) we get $\left(\alpha_{0,0}^{ \pm}=1\right)$

$$
\begin{gathered}
\alpha_{2 q, 0}^{+}=-\frac{1}{8 \varepsilon_{m+2 q-2} q(m+q)} \alpha_{2 q-2,0}^{+}, \quad m \geq 0, q \geq 1, \\
\alpha_{2 q, 0}^{-}=\frac{1}{16 q(m-q)} \alpha_{2 q-2,0}^{-}, \quad m \geq 2 q, q \geq 1 .
\end{gathered}
$$

From (35) we easily find that

$$
\begin{gathered}
\alpha_{2 q, 0}^{+}=(-1)^{q} \frac{2 m !}{\varepsilon_{m} 16^{q} q !(m+q) !}, \quad m \geq 0, q \geq 1, \\
\alpha_{2 q, 0}^{-}=\frac{(m-q-1) !}{16^{q} q !(m-1) !}, \quad m \geq 2 q, q \geq 0 .
\end{gathered}
$$

From (9), (11)-(13), (17), (20a) and (36a), we obtain

$$
\alpha_{2 q, 2}^{+}=\left\{\begin{array}{l}
-\frac{1}{1024}, \quad m=1, q=1, \\
-\frac{q}{16(q-1)(q+1)^{2}} \alpha_{2 q-2,2}^{+}, \quad m=1, \quad q \geq 2, \\
0, \quad m \neq 1, \quad q \geq 1 .
\end{array}\right.
$$

From (37), as well as from (17), (20a) and (36a), we obtain, for $q \geq 0$,

$$
\alpha_{2 q, 2}^{+}=\left\{\begin{array}{l}
(-1)^{q} \frac{q}{16^{q+1}[(q+1) !]^{2}}, \quad m=1, \\
0, \quad m \neq 1 .
\end{array}\right.
$$

Using (17), (20b) and (36b), we get, for $q \geq 1$,

$$
\alpha_{2 q, 2}^{-}=\left\{\begin{array}{l}
\frac{1}{16^{q+1} q(q+1)(2 q) !}, \quad m=2 q+1, \\
0, \quad m \neq 2 q+1 .
\end{array}\right.
$$


Using (21) for $k=2$ with (34) and (36), we easily find $\alpha_{2 q, 4}^{+}$and $\alpha_{2 q, 4}^{-}$, respectively:

$$
\alpha_{2 q, 4}^{+}=\left\{\begin{array}{l}
(-1)^{q} \frac{4 q}{16^{q+3}+(q+2) !}, \quad m=1, \\
(-1)^{q} \frac{q\left(47 q^{2}+222 q+247\right)}{16^{q+2} 18(q+2) !(q+3) !}, \quad m=2, \\
(-1)^{q} \frac{2 q\left(m^{2}+3 m+m q+3 q+4\right) m !}{\varepsilon_{m} 16^{q+2}\left(m^{2}-1\right)(m+1)(q+1) !(m+q+1) !}, \quad m=0 \text { or } m \geq 3
\end{array}\right.
$$

for $q \geq 0$, and, for $q \geq 1$,

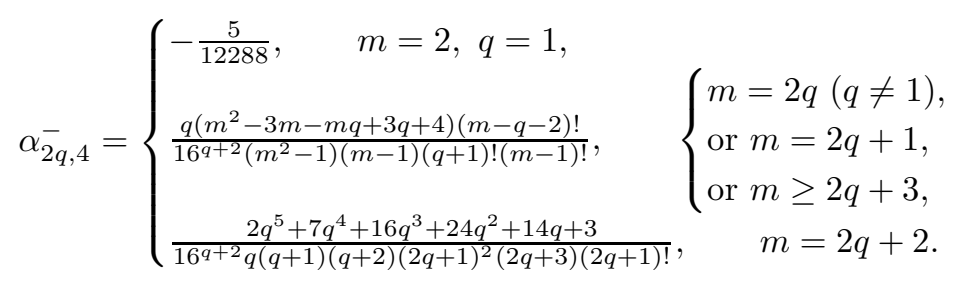

The analytic evaluation of $v_{2 k}^{ \pm}(2 q)$ becomes more and more lengthy and tedious, as $k$ increases (see (25)). After some manipulation we can obtain from (25) the analytic expressions for $v_{6}^{ \pm}(2 q)(k=3)$ which are given in the Appendix.

The explicit values of the $B$ 's depend on the normalization used. Here it will be assumed [14] that $\operatorname{Se}_{m}(h, 1)=1$, or equivalently,

$$
\sum_{n=0}^{\infty} B_{n}^{e}(h, m)=1
$$

with $n$ and $m$ both even or both odd. Substituting (40) into (40), we obtain

$$
B_{m}^{e}(h, m)\left[1+\sum_{q=1}^{\infty} \sum_{k=0}^{\infty}\left(\alpha_{2 q, 2 k}^{+}+\alpha_{2 q, 2 k}^{-}\right) h^{2 q+2 k}\right]=1 .
$$

The expansion of $B_{m}^{e}(h, m)$ for small values of $h$ has the expression

$$
B_{m}^{e}(h, m)=1+g_{2} h^{2}+g_{4} h^{4}+g_{6} h^{6}+\cdots .
$$

Substituting (42) into (41), we carry out the various multiplications and equate the coefficients of like powers of $h$, calculating successively the coefficients $g_{2 s}$, which are independent of $h$ and given by the formula

$$
g_{2 s}=-\sum_{q=1}^{s} \sum_{k=0}^{s-q} g_{2 k}\left[\alpha_{2 q, 2(s-q-k)}^{+}+\alpha_{2 q, 2(s-q-k)}^{-}\right], \quad s=1,2, \ldots,
$$

while $g_{0}=1$.

Using, finally, (4) and (42) in (2), we obtain, after some manipulation, the power series expansion for $\operatorname{Se}_{m}(h, \cos \theta)$ :

$$
\begin{aligned}
\operatorname{Se}_{m}(h, \cos \theta)=\sum_{l=0}^{\infty} g_{2 l} h^{2 l}\left\{\cos m \theta+\sum_{q=1}^{\infty}\right. & \sum_{k=0}^{\infty}\left\{\alpha_{2 q, 2 k}^{+} \cos [(m+2 q) \theta]\right. \\
& \left.\left.+\alpha_{2 q, 2 k}^{-} \cos [(m-2 q) \theta]\right\} h^{2(q+k)}\right\} .
\end{aligned}
$$

We recall that in each case $\alpha_{2 q, 2 k}^{-}=0$, if $0 \leq m<2 q$. 
The above-calculated expansion coefficients are also useful for the evaluation of the even radial Mathieu functions of any kind. These functions are solutions of the equation

$$
\frac{d^{2} y}{d \mu^{2}}-\left(b-\frac{h^{2}}{2} \cosh 2 \mu\right) y=0,
$$

which is merely (10) with $\theta=i \mu$ (in this case $b=b e_{m}$ ), and are expressed as [14]

$$
\mathrm{Ze}_{m}(h, \cosh \mu)=\sqrt{\frac{\pi}{2}} \sum_{n=0}^{\infty}(-1)^{(n-m) / 2} B_{n}^{e}(h, m) Z_{n}(h \cosh \mu) .
$$

Here $Z_{n}$ is the cylindrical Bessel function of any kind and $\mathrm{Ze}_{m}$ the corresponding even radial Mathieu function of the same kind. By using (4), (42) and (43) in (46) we can find its expansion for small values of $h$.

\section{Calculation of the expansion Coefficients FOR THE ODD FUNCTIONS}

The odd angular Mathieu functions of integral order are given by the series [13- 15

$$
\operatorname{So}_{m}(h, \cos \theta)=\sum_{n=1}^{\infty} B_{n}^{o}(h, m) \sin n \theta, \quad m \geq 1
$$

where $n$ and $m$ are both even or both odd. Substituting (47) into (11), with $b=$ $b o_{m}$, we obtain the following second order recurrence relation for the expansion coefficients $B_{n}^{o}(h, m)$ [13]-15], [17:

$$
\left(-n^{2}+b o_{m}\right) B_{n}^{o}-\frac{h^{2}}{4}\left[B_{n+2}^{o}+B_{n-2}^{o}-\left(2-\varepsilon_{n-1}\right) B_{n}^{o}\right]=0, \quad m, n \geq 1,
$$

where $B_{-n}^{o}=0$ for $n \geq 0$.

The expansions (4) and (5) are also valid in this case, with $e$ replaced by $o$. Now, in (44), $m \geq 1$ for the upper ( + ) sign and $m \geq 2 q+1$ for the lower $(-)$ sign.

Following steps identical to those for the even functions, we obtain, with $n=$ $m \geq 1$, in place of (6) and (7), the relations

$$
l_{2}=-\left(2-\varepsilon_{m-1}\right) / 4
$$

and

$$
\frac{1}{4}\left(\alpha_{2,2 k}^{+}+\alpha_{2,2 k}^{-}\right)=l_{2 k+4}, \quad k=0,1,2, \ldots .
$$

Setting next $n=m \pm 2 q$, where $q \geq 1$ ( $m \geq 2 q+1$ for the lower sign), in (48) and using (4) and (5) with $e$ replaced by $o$, we again obtain (8)-(14), with the only differences that $\varepsilon_{m+2 q-2}$ in $f^{+}$(equation (12)) and $\varepsilon_{m-2 q-2}$ in $p^{-}$(equation (14)) should be replaced by 2 , and that $g^{ \pm}$(equation (13)) should change their sign. For this reason, (15)-(19) and (21)-(28) remain the same (the remarks after (28) for $\delta_{2 k}^{-}(2 q)$ are also valid in this case, with $2 q+2,2 q+1$ and $2 q$ replaced by $2 q+3$, $2 q+2$ and $2 q+1$, respectively) while $v_{2}^{ \pm}(2 q)$ in (20) simply change their sign. So, in this case,

$$
v_{2}^{+}(2 q)=\left\{\begin{array}{l}
-\frac{q}{16(q+1)}, \quad m=1 \\
0, \quad m \neq 1,
\end{array}\right.
$$


for $q \geq 0$, and, for $q \geq 1$,

$$
v_{2}^{-}(2 q)=\left\{\begin{array}{l}
-\frac{1}{16 q(q+1)}, \quad m=2 q+1, \\
0, \quad m \neq 2 q+1,
\end{array}\right.
$$

while the expansion coefficients $l_{2 j}(2 \leq j \leq k)$ should be calculated from (50) (with the use of $\alpha_{2,2 j-4}^{ \pm}$calculated from (21) with $q=1$ ). Their expressions for $j \leq 8$ can also be found in [13, [15, 17].

Setting $k=2$ in (25), we again obtain (29) $-(32 \mathrm{a})$, with the only differences that now $m \geq 1$ for the superscript + and $m \geq 2 q+1$ of the superscript - . Equations (32b) are replaced by

$$
\delta_{4}^{-}(2 q)= \begin{cases}\frac{1}{256} \sum_{i=1}^{q} \frac{1}{i(i+1)(i-m)(i-m+1)}, & m \geq 2 q+3, \\ \frac{1}{256} \sum_{i=1}^{q-1} \frac{1}{i(i+1)(i-m)(i-m+1)}, & m=2 q+1 \text { or } 2 q+2, q \neq 1, \\ 0, \quad q=1, m=3 \text { or } 4 . & \end{cases}
$$

Substituting the aforementioned equations in (29) and using the expressions for $l_{4}$ found from (50) and (8) with $k=0$ and $q=1$, respectively,

$$
l_{4}=\left\{\begin{array}{l}
-1 / 128, \quad m=1 \\
-1 / 192, \quad m=2, \\
1 /\left[32\left(m^{2}-1\right)\right], \quad m \geq 3,
\end{array}\right.
$$

we obtain, after some manipulation, the results

$$
v_{4}^{+}(2 q)=\left\{\begin{array}{l}
\frac{q}{1024(q+2)}, \quad m=1, \\
-\frac{q(7 q+23)}{9216(q+2)(q+3)}, \quad m=2, \\
\frac{q\left(m^{2}+3 m+m q+3 q+4\right)}{256\left(m^{2}-1\right)(m+1)(q+1)(m+q+1)}, \quad m \geq 3,
\end{array}\right.
$$

for $q \geq 0$, and, for $q \geq 1$,

$$
v_{4}^{-}(2 q)=\left\{\begin{array}{lc}
\frac{q\left(m^{2}-3 m-m q+3 q+4\right)}{256\left(m^{2}-1\right)(m-1)(q+1)(m-1-q)}, & m \neq 2 q+2, \\
\frac{2 q^{5}+7 q^{4}-16 q^{2}-14 q-3}{256 q(q+1)^{2}(q+2)(2 q+1)^{2}(2 q+3)}, & m=2 q+2 .
\end{array}\right.
$$

From (8), (11), (12) (keeping in mind the modification for $f^{+}$) we have in this case $\left(\alpha_{0,0}^{ \pm}=1\right)$

$$
\alpha_{2 q, 0}^{ \pm}=\mp \frac{1}{16 q(m \pm q)} \alpha_{2 q-2,0}^{ \pm}, \quad\left\{\begin{array}{c}
m \geq 1 \text { (upper sign) } \\
m \geq 2 q+1 \text { (lower sign) }
\end{array}\right\}, \quad q \geq 1 .
$$

From this we easily obtain

$$
\begin{aligned}
& \alpha_{2 q, 0}^{+}=(-1)^{q} \frac{m !}{16^{q} q !(m+q) !}, \quad m \geq 1, q \geq 0, \\
& \alpha_{2 q, 0}^{-}=\frac{(m-q-1) !}{16^{q} q !(m-1) !}, \quad m \geq 2 q+1, q \geq 0 .
\end{aligned}
$$


From (9), (11)-(13), (17), (51a) and (56a), keeping in mind the aforementioned modifications, we find that

$$
\alpha_{2 q, 2}^{+}=\left\{\begin{array}{l}
\frac{1}{1024}, \quad m=1, q=1, \\
-\frac{q}{16(q-1)(q+1)^{2}} \alpha_{2 q-2,2}^{+}, \quad m=1, q \geq 2, \\
0, \quad m \neq 1, \quad q \geq 1 .
\end{array}\right.
$$

From this and from (17), (51a) and (56a), we obtain

$$
\alpha_{2 q, 2}^{+}=\left\{\begin{array}{l}
(-1)^{q+1} \frac{q}{16^{q+1}[(q+1) !]^{2}}, \quad m=1, \\
0, \quad m \neq 1,
\end{array} \quad(q \geq 0) .\right.
$$

Using (17), (51b) and (56b), we get

$$
\alpha_{2 q, 2}^{-}=\left\{\begin{array}{l}
-\frac{1}{16^{q+1} q(q+1)(2 q) !}, \quad m=2 q+1, \\
0, \quad m \neq 2 q+1,
\end{array} \quad(q \geq 1) .\right.
$$

From (21) with $k=2$ and (54), (56) we easily obtain $\alpha_{2 q, 4}^{+}$and $\alpha_{2 q, 4}^{-}$:

$$
\alpha_{2 q, 4}^{+}=\left\{\begin{array}{l}
(-1)^{q} \frac{4 q}{16^{q+3} q !(q+2) !}, \quad m=1, \\
(-1)^{q+1} \frac{q(q+1)(7 q+23)}{16^{q+2} \cdot 18(q+2) !(q+3) !}, \quad m=2, \\
(-1)^{q} \frac{q\left(m^{2}+3 m+m q+3 q+4\right) m !}{16^{q+2}\left(m^{2}-1\right)(m+1)(q+1) !(m+q+1) !}, \quad m \geq 3,
\end{array}\right.
$$

for $q \geq 0$, and, for $q \geq 1$,

$$
\alpha_{2 q, 4}^{-}=\left\{\begin{array}{l}
\frac{q\left(m^{2}-3 m-m q+3 q+4\right)(m-q-2) !}{16^{q+2}\left(m^{2}-1\right)(m-1)(q+1) !(m-1) !}, \quad m \neq 2 q+2, \\
\frac{2 q^{5}+7 q^{4}-16 q^{2}-14 q-3}{16^{q+2} q(q+1)(q+2)(2 q+1)^{2}(2 q+3)(2 q+1) !}, \quad m=2 q+2 .
\end{array}\right.
$$

As in Section 2, the analytic evaluation of $v_{2 k}^{ \pm}(2 q)$ becomes lengthier and more tedious as $k$ increases. By using (25), we have found also the analytic expressions for $v_{6}^{ \pm}(2 q)(k=3)$ in the case of the odd functions. These expressions are given in the Appendix.

The normalization used for the calculation of the explicit values of $B$ 's is 14 $d \operatorname{So}_{m}(h, 1) / d \theta=1$, or, equivalently,

$$
\sum_{n=1}^{\infty} n B_{n}^{o}(h, m)=1
$$

with $n$ and $m$ both even or both odd. Substituting (4) (with $e$ replaced by $o$ ) into (60), we obtain

$$
B_{m}^{o}(h, m)\left\{m+\sum_{q=1}^{\infty} \sum_{k=0}^{\infty}\left[(m+2 q) \alpha_{2 q, 2 k}^{+}+(m-2 q) \alpha_{2 q, 2 k}^{-}\right] h^{2 q+2 k}\right\}=1 .
$$

The expansion of $B_{m}^{o}(h, m)$ for small values of $h$ has the expression

$$
B_{m}^{o}(h, m)=\frac{1}{m}+g_{2} h^{2}+g_{4} h^{4}+g_{6} h^{6}+\cdots .
$$


Substituting (62) into (61), we follow the same procedure as for the even functions, and finally get

$g_{2 s}=-\frac{1}{m} \sum_{q=1}^{s} \sum_{k=0}^{s-q} g_{2 k}\left[(m+2 q) \alpha_{2 q, 2(s-q-k)}^{+}+(m-2 q) \alpha_{2 q, 2(s-q-k)}^{-}\right], \quad s=1,2, \ldots$, with $g_{0}=1 / m$.

$\operatorname{So}_{m}(h, \cos \theta)$ is given by (44), with $\cos$ replaced by $\sin$. We recall that in each case $\alpha_{2 q, 2 k}^{-}=0(k \geq 0)$ if $1 \leq m \leq 2 q+1$.

The evaluation of the odd radial Mathieu functions $\mathrm{Zo}_{m}(h, \cosh \mu)$ of any kind, which are solutions of (45) with $b=b o_{m}$, is made, in analogy to (46), by the formula [14]

$$
\mathrm{Zo}_{m}(h, \cosh \mu)=\sqrt{\frac{\pi}{2}} \tanh \mu \sum_{n=1}^{\infty}(-1)^{(n-m) / 2} n B_{n}^{o}(h, m) Z_{n}(h \cosh \mu) .
$$

\section{Relations AMONG the EXPANSION COEFFicients FOR EVEN AND ODD FUNCTIONS}

In this section we shall use the superscripts e and o to distinguish the various expansion coefficients used for the even and odd functions.

By comparing the expansion coefficients $l_{2 j}^{\mathrm{e}(\mathrm{o})}$, given in [13, [15], [17] for $j \leq 8$, we can easily find the following relations connecting them (their validity for general $j$ will be proved at the end of this section):

$$
\begin{array}{lll}
l_{2 j}^{\mathrm{e}}=l_{2 j}^{\mathrm{o}}=0, & j \text { odd } & \left\{\begin{array}{l}
m \geq 0(m \text { even }) \\
m>j(m \text { odd })
\end{array}\right\}, \\
l_{2 j}^{\mathrm{e}}=(-1)^{j} l_{2 j}^{\mathrm{o}}, & m \text { odd, } \\
l_{2 j}^{\mathrm{e}}=l_{2 j}^{\mathrm{o}}, & j \text { even }<m \text { even, } &
\end{array}
$$

From (20a), (51a), (34a), (54a), as well as (A1) and its analog for odd functions given in the Appendix, we observe that for $k=1,2,3$

$$
v_{2 k}^{\mathrm{o}+}(2 q)=(-1)^{k} v_{2 k}^{\mathrm{e}+}(2 q), \quad\left\{\begin{array}{l}
m=1,3, \ldots, k(k \text { odd }), \\
m \neq 2,4, \ldots, k(k \text { even }),
\end{array}\right.
$$

while for $k$ odd and $m$ even or $m$ odd $>k$, (66) is also valid, but with both $v$ 's equal to zero, as will be proved later in this section. We suppose now that (66) is valid for $k=1,2, \ldots, r$, and we shall prove it for $k=r+1$. We use (25), with $k=r+1$, for odd functions:

$$
\begin{aligned}
v_{2 r+2}^{\mathrm{o}+}(2 q)=\sum_{i=1}^{q}\left[g^{\mathrm{o}+}(2 i) v_{2 r}^{\mathrm{o}+}(2 i)+\right. & t^{\mathrm{o}+}(2 i) \sum_{j=2}^{r+1} l_{2 j}^{\mathrm{o}} v_{2 r+2-2 j}^{\mathrm{o}+}(2 i) \\
& \left.+p^{\mathrm{o}+}(2 i+2) v_{2 r-2}^{\mathrm{o}+}(2 i+2) f^{\mathrm{o}+}(2 i)\right] .
\end{aligned}
$$

Using the relations $g^{\mathrm{o}+}=-g^{\mathrm{e}+}, t^{\mathrm{o}+}=t^{\mathrm{e}+}, p^{\mathrm{o}+}=p^{\mathrm{e}+}, f^{\mathrm{o}+}=f^{\mathrm{e}+}(m \geq 1)$, as well as (65) and (66) in (67), we very easily prove (66) for $k=r+1$ and $m$ odd ( $r$ even or odd). If $m$ is even, $g^{\mathrm{o}+}=g^{\mathrm{e}+}=0$; in this case for $j$ odd, $l_{2 j}^{\mathrm{o}}=l_{2 j}^{\mathrm{e}}=0$, while for $j$ even $l_{2 j}^{\mathrm{o}}=l_{2 j}^{\mathrm{e}}$, if $j \leq r+1<m$ (see (65)). Using (66), as 
well as the aforementioned relations in (67), we also prove (66) for $k=r+1$ and $m=$ even $>r+1$ ( $r$ even or odd $)$.

From (20b), (51b), (34b), (54b), as well as (A2) and its analog for odd functions given in the Appendix, we observe that for $k=1,2,3$

$$
v_{2 k}^{\mathrm{o}-}(2 q)=(-1)^{k} v_{2 k}^{\mathrm{e}-}(2 q), \quad\left\{\begin{array}{l}
m=2 q+1,2 q+3, \ldots, 2 q+k(k \text { odd }), \\
m \neq 2 q+2,2 q+4, \ldots, 2 q+k(k \text { even }),
\end{array}\right.
$$

while for $k$ odd and $m$ even $(>2 q+1)$, or $m$ odd $(>2 q+k)$, (68) is also valid, with both $v$ 's equal to zero, as will be proved in what follows.

Following the same procedure as in the previous case (with the superscript + replaced by -), we prove (68) very easily for $k=r+1$ when $m$ is odd, or when $m$ is even $>2 q+r+1$ ( $r$ even or odd in both cases).

Next we examine the relation

$$
v_{2 k}^{\mathrm{e} \pm}(2 q)=0 \quad k \text { odd, } \quad\left\{\begin{array}{l}
m \text { even or } m \text { odd }>k(+), \\
m \text { even or } m \text { odd }>2 q+k(-),
\end{array}\right.
$$

which is valid for $k=1,3$ (equations (20) and (A1), (A2)). By supposing that it is also valid for $k=1,3, \ldots, r$, with $r$ odd, we shall prove it for $k=r+2$.

Substituting in (25), we obtain

$$
\begin{aligned}
v_{2 r+4}^{\mathrm{e} \pm}(2 q)=\sum_{i=1}^{q}\left[g^{\mathrm{e} \pm}(2 i) v_{2 r+2}^{\mathrm{e} \pm}(2 i)+\right. & t^{\mathrm{e} \pm}(2 i) \sum_{j=2}^{r+2} l_{2 j}^{\mathrm{e}} v_{2 r+4-2 j}^{\mathrm{e} \pm}(2 i) \\
& \left.+p^{\mathrm{e} \pm}(2 i+2) v_{2 r}^{\mathrm{e} \pm}(2 i+2) f^{\mathrm{e} \pm}(2 i)\right] .
\end{aligned}
$$

Using (13), (65a) and (69) in (70), we very easily prove (69) for $k=r+2$ and $m$ even, as well as for $m$ odd, with $m>r+2(+)$ or $m>2 q+r+2(-)$.

Finally, for $v_{2 k}^{\mathrm{o} \pm}(2 q)$, equations (69) and (70) , as well as the aforementioned proof, remain the same, with the only difference that e is replaced by o.

Relations (66), (68), as well as (69) and its corresponding one for odd functions, remain valid if we replace $v_{2 k}(2 q)$ (with the proper superscripts in each case) by the corresponding $\alpha_{2 q, 2 k}$ (see (21)), because $\alpha_{2 q, 0}^{\mathrm{e}+}=\alpha_{2 q, 0}^{\mathrm{o}+}$ for $m \geq 1$ (by (36a), (56a)) and $\alpha_{2 q, 0}^{\mathrm{e}_{-}}=\alpha_{2 q, 0}^{\mathrm{o}-}$ for $m \geq 2 q+1$ (by (36b)), (56b)).

Now we turn to the proof of the relations (65) for general $j$. We suppose that they are valid for $j=1,2, \ldots, r$, and we shall prove them for $j=r+1$. Setting $k+2=r+1(k=r-1)$ in (7) and (50), we obtain, respectively,

$$
\begin{gathered}
l_{2 r+2}^{\mathrm{e}}=\frac{1}{4}\left(\alpha_{2,2 r-2}^{\mathrm{e}+}+\frac{2}{\varepsilon_{m-2}} \alpha_{2,2 r-2}^{\mathrm{e}-}\right), \\
l_{2 r+2}^{\mathrm{o}}=\frac{1}{4}\left(\alpha_{2,2 r-2}^{\mathrm{o}+}+\alpha_{2,2 r-2}^{\mathrm{o}-}\right) .
\end{gathered}
$$

If we use the remarks in the paragraph before (171), we get

$$
l_{2 r+2}^{\mathrm{e}}=\frac{(-1)^{r-1}}{4}\left(\alpha_{2,2 r-2}^{\mathrm{o}+}+\frac{2}{\varepsilon_{m-2}} \alpha_{2,2 r-2}^{\mathrm{o}-}\right),
$$

with the limitations given for (66) and (68). By comparing (72) and (73), with the use of these limitations, we prove (65) for $j=r+1$ ( $r$ even or odd). 


\section{Comparisons With EXISTing DATA}

In this section we discuss various checks and comparisons made to confirm the correctness of our results.

Formulas (36) and (56) are the same as the corresponding ones in [13, 15], [17] for each $q$ and $m$. In the special cases with $m=0-2,(38),(58)$, as well as (39), (59) give the results found in [13], [15], 16]. The orders of the first terms omitted there can also be confirmed by the use of (A1), A2 and the analogous formulas for odd functions.

Using (21), (36), (38), (39) and (A1), (A2), all with $q=1$, in (7) we obtain, for each value of $m$, the expansion coefficients $l_{4}, l_{6}, l_{8}$ and $l_{10}$ (with $k=0,1,2$ and 3 , respectively) of the characteristic values $b e_{m}$, which are also given in [13], [15], [17. The same is valid also for $b o_{m}$, by using (21), (56), (58), (59) and the corresponding versions of (A1), (A2) for odd functions, in (50). For $m=0, \ldots, 4$ our coefficients are the same as the ones given in [26], 27].

Finally, by using (43) and (44) we verify the expansions for $\mathrm{Se}_{m}$ in the special cases with $m=0, \ldots, 4$ examined in 14, [26, 27. The same is valid also for $\mathrm{So}_{m}$ $(m=1, \ldots, 4)$ 14, 26], 27] by virtue of (63) and the formula corresponding to (44) for odd functions.

It is obvious that our results are much more general than the ones used above for the confirmation of their validity. The latter constitute special cases of our formulas, for $m=0, \ldots, 4$ (with the exception of those corresponding to $l_{2 k+4}$, $k=0, \ldots, 3$, which are valid for each $m$ ).

It should be noticed here that (5) can also be used for the evaluation of the initial estimates for $b e_{m}$ and $b o_{m}$, in the iterative algorithms described in [25] for their computation.

\section{APPENDIX}

The analytic expressions for $v_{6}^{ \pm}(2 q)(k=3)$ obtained from (25) after some manipulation are the following.

1) Even functions. For $q \geq 0$ we have

$$
v_{6}^{+}(2 q)=\left\{\begin{array}{l}
-\frac{q(3 q+8)}{32768(q+2)^{2}}, \quad m=1, \\
\frac{q\left(11 q^{2}+48 q+49\right)}{294912(q+1)(q+2)(q+3)}, \quad m=3 \\
0, \quad m \neq 1,3 .
\end{array}\right.
$$

Moreover,

$$
v_{6}^{-}(2 q)=\left\{\begin{array}{l}
-\frac{1}{32768}, \quad q=1, \quad m=3, \\
\frac{q^{2}-q+2}{16384\left(q^{2}-1\right) q^{3}(q+2)}, \quad m=2 q+1, \quad q \geq 2, \\
\frac{1}{4096 q(q+1)^{2}(q+2)^{2}(q+3)}, \quad m=2 q+3, q \geq 1, \\
0, \quad \text { otherwise. }
\end{array}\right.
$$

For the evaluation of (A1) and (A2) we have used $l_{4}$ from (33), while

$$
l_{6}=\left\{\begin{array}{l}
-1 / 4096, \quad m=1, \\
1 / 4096, \quad m=3, \\
0, \quad m \neq 1,3
\end{array}\right.
$$

calculated from (7) and (38) with $q=1$. 
2) Odd functions. For the odd functions, $v_{6}^{+}(2 q), v_{6}^{-}(2 q)$ and $l_{6}$ are opposite to the corresponding ones for the even functions given in (A1)- (A3).

\section{REFERENCES}

[1] J. H. Richmond, Scattering by a conducting elliptic cylinder with dielectric coating, Radio Sci. 23 (1988), 1061-1066.

[2] R. Holland and V. P. Cable, Mathieu functions and their applications to scattering by a coated strip, IEEE Trans. Electromagn. Compat. 34 (1992), 9-16.

[3] T. M. Habashy, J. A. Kong and W. C. Chew, Scalar and vector Mathieu transform pairs, J. Appl. Phys. 60 (1986), 3395-3399.

[4] F. A. Alhargan and S. R. Judah, Frequency response characteristics of multiport planar elliptic patch, IEEE Trans. Microwave Theory Tech. 40 (1992), 1726-1730.

[5] D. W. Jordan and P. Smith, Nonlinear Ordinary Differential Equations, Clarendon Press, Oxford, 1987. MR 89a:34001

[6] J. E. Lewis and G. Deshpande, Models on elliptical cross-section dielectric-tube waveguides, IEE J. Microwaves, Optics and Acoustics 3 (1979), 147-155.

[7] S. Caorsi, M. Pastorino and M. Raffetto, Electromagnetic scattering by a multilayer elliptic cylinder under transverse-magnetic illumination: series solution in terms of Mathieu functions, IEEE Trans. Antennas Propagat., 45 (1997), 926-935.

[8] N. B. Kakogiannos and J. A. Roumeliotis, Electromagnetic scattering from an infinite elliptic metallic cylinder coated by a circular dielectric one, IEEE Trans. Microwave Theory Tech. 38 (1990), 1660-1666.

[9] J. A. Roumeliotis, H. K. Manthopoulos and V. K. Manthopoulos, Electromagnetic scattering from an infinite circular metallic cylinder coated by an elliptic dielectric one, IEEE Trans. Microwave Theory Tech. 41 (1993), 862-869.

[10] J. A. Roumeliotis and S. P. Savaidis, Cutoff frequencies of eccentric circular-elliptic metallic waveguides, IEEE Trans. Microwave Theory Tech. 42 (1994), 2128-2138.

[11] _ Scattering by an infinite circular dielectric cylinder coating eccentrically an elliptic metallic one, IEEE Trans. Antennas Propagat. 44 (1996), 757-763.

[12] S. P. Savaidis and J. A. Roumeliotis, Scattering by an infinite elliptic dielectric cylinder coating eccentrically a circular metallic or dielectric cylinder, IEEE Trans. Microwave Theory Tech. 45 (1997), 1792-1800.

[13] N. W. McLachlan, Theory and Application of Mathieu Functions, Clarendon, Oxford, 1947. MR 9:31b

[14] P. M. Morse and H. Feshbach, Methods of Theoretical Physics, McGraw-Hill, New York, 1953. MR 15:583h

[15] J. Meixner and F. W. Schafke, Mathieusche Funktionen und Sphäroidfunktionen, SpringerVerlag, Berlin, 1954. MR 16:586g

[16] E. T. Whittaker and G. N. Watson, A Course of Modern Analysis, 4th ed., reprinted, Cambridge Univ. Press, 1996. MR 97k:01072

[17] M. Abramowitz and I. A. Stegun, Handbook of Mathematical Functions, Dover, New York, 1972. MR 85j:00005a (reprint)

[18] R. B. Shirts, The computation of eigenvalues and solutions of Mathieu's differential equation for noninteger order, ACM Trans. Math. Software 19 (1993), 377-390.

[19] — Algorithm 721 MTIEU1 and MTIEU2: Two subroutines to compute eigenvalues and solutions to Mathieu's differential equation for noninteger and integer order, ACM Trans. Math. Software 19 (1993), 391-406.

[20] N. Toyama and K. Shogen, Computation of the value of the even and odd Mathieu functions of order $N$ for a given parameter $S$ and an argument $X$, IEEE Trans. Antennas Propagat. 32 (1984), 537-539. MR 85j:33006

[21] Delft Numerical Analysis Group, On the computation of Mathieu functions, J. Engrg. Math. 7 (1973), 39-61. MR 51:3579

[22] S. R. Rengarajan and J. E. Lewis, Mathieu functions of integral orders and real arguments, IEEE Trans. Microwave Theory Tech. 28 (1980), 276-277.

[23] D. S. Clemm, Characteristic values and associated solutions of Mathieu's differential equation, Comm. Assoc. Comput. Mach. 12 (1969), 399-407. 
[24] W. R. Leeb, Algorithm 537: Characteristic values of Mathieu's differential equation, ACM Trans. Math. Software 5 (1979), 112-117.

[25] F. Alhargan, A complete method for the computations of Mathieu characteristic numbers of integer orders, SIAM Rev. 38 (1996), 239-255. MR 97h:33036

[26] H. Rubin, Anecdote on power series expansions of Mathieu functions, J. Math. and Phys. 43 (1964), 339-341. MR 30:287

[27] R. Barakat, A. Houston and E. Levin, Power series expansions of Mathieu functions with tables of numerical results, J. Math. and Phys. 42 (1963), 200-247. MR 27:4966

[28] G. C. Kokkorakis and J. A. Roumeliotis, Acoustic eigenfrequencies in concentric spheroidalspherical cavities, J. Sound. Vibr. 206 (1997), 287-308.

Department of Electrical and Computer Engineering, National Technical University of Athens, Athens 15773, Greece

Department of Electrical and Computer Engineering, National Technical UniverSity of Athens, Athens 15773, Greece

E-mail address: iroumel@cc.ece.ntua.gr 